\title{
ON LINEAR VOLTERRA INTEGRAL EQUATIONS OF CONVOLUTION TYPE
}

\author{
JOHN S. LEW
}

\begin{abstract}
Let $\boldsymbol{A}$ be the set of all complex-valued locally integrable functions defined on $[0,+\infty)$, and let $T$ be the topology for $A$ determined by the seminorms $t_{r}(f)=\int_{0}^{r}|f(x)| d x$ for $r=1$, $2, \cdots$, so that $A$ is a topological algebra under pointwise addition, complex scalar multiplication, and Laplace convolution. Then the map $f \rightarrow f^{\prime}$ from each element to its quasi-inverse is a homeomorphism of $(A, T)$ onto itself. For each $f, g$ in $A$ the equation $v=f+$ $g * v$ has a unique solution in $A$ which depends $T$-continuously on $f, g$, and is the $T$-limit of Picard approximations. The set of all $f$ in $A$ with $f^{\prime}$ in $L^{1}[0,+\infty)$ is a set of first category in $(A, T)$ but an open subset of $A$ with the metric $\|f-g\|_{1}$. For each series $\sum_{n=1}^{\infty} p_{n} z^{n}$ converging in some neighborhood of $z=0$, and each element $f$ in $A$, the series $\sum_{n=1}^{\infty} p_{n} f^{* n}$ converges in $T$ to some element $p^{*}(f)$ in $A$.
\end{abstract}

1. Introduction. In the standard development for a linear Volterra integral equation of the second kind

$$
v(x)=f(x)+\int_{0}^{x} k(x, y) v(y) d y,
$$

a sequence of approximations is constructed by Picard's method of iteration, and the solution of (1.1) is obtained as the limit of this sequence. To insure the validity of this argument, the kernel $k$ is traditionally assumed continuous [Lovitt (1924), p. 14] or at least assumed square-integrable on bounded sets [Tricomi (1957), p. 10]. However if the kernel is of convolution type, that is, if

$$
k(x, y)=g(x-y),
$$

then the right side of $(1.1)$ is well-defined under more general hypotheses. Indeed even if $f$ and $g$ are no more than locally integrable, then still a solution $v$ can be shown to exist [e.g. Miller and Feldstein (1971), Lemma 1], though somewhat less directly.

Received by the editors August 20, 1971 and, in revised form, February 6, 1972. AMS 1970 subject classifications. Primary 45A05, 45D05; Secondary 45E10, 45Lxx.

Key words and phrases. Linear Volterra integral equation, convolution kernel, locally integrable function, quasi-inverse, Picard iteration, integrable resolvent. 
We propose to note a few properties of such solutions. From basic results for the algebra $A$ of all locally integrable functions, furnished with a suitable vector topology, we obtain simply and independently the existence of a solution for (1.1)-(1.2) and its continuity in $f$ and $g$. We then use these results to study in $A$ the convergence of Picard iteration, the development of a functional calculus, and the set of kernels which have integrable resolvents.

2. The algebra $A$. Let $A$ denote the set of all complex-valued locally integrable functions on $[0,+\infty)$, and for each $f, g$ in $A$ let $f * g$ denote their Laplace convolution, given by

$$
[f * g](x)=\int_{0}^{x} f(x-y) g(y) d y .
$$

It is clear that $A$ is closed under pointwise addition and scalar multiplication; it is well known that $A$ is closed under Laplace convolution. Indeed $A$ with these compositions is a commutative associative algebra over the complex numbers [Mikusiński (1959), pp. 345-349]. Thus positive integral *-powers can be defined for any $f$ in $A$ :

$$
f^{* 1}=f, \quad f^{* n+1}=f * f^{* n} \text { for } n=1,2, \cdots .
$$

For $r=1,2, \cdots$ and any $f$ in $A$ we let

$$
\begin{aligned}
t_{r}(f) & =\int_{0}^{r}|f(x)| d x, \\
u_{r}(f) & =\operatorname{ess} \sup \{|f(x)|: 0 \leqq x<r\},
\end{aligned}
$$

and we admit the value $+\infty$ for $u_{r}(f)$. Then the families $\left\{t_{r}\right\}$ and $\left\{u_{r}\right\}$ of seminorms [Schaefer (1966), p. 39] generate the topologies $T$ and $U$ respectively in $A$, corresponding to $L^{1}$ and $L^{\infty}$ convergence on bounded sets. Obviously convergence in $U$ implies convergence in $T$. For both topologies the space $A$ is complete, metrizable, and locally convex [Schaefer (1966), p. 28], while the maps $(c, f) \rightarrow c f,(f, g) \rightarrow f+g,(f, g) \rightarrow$ $f * g$ are jointly continuous, the last through Fubini's theorem and direct estimates, which yield the inequalities

$$
t_{r}(f * g) \leqq t_{r}(f) t_{r}(g), \quad u_{r}(f * g) \leqq t_{r}(f) u_{r}(g) \leqq r u_{r}(f) u_{r}(g)
$$

If $f$ and $g$ are elements of $A$ which satisfy

$$
f+g=f * g
$$

then each is called a quasi-incerse of the other. We can always adjoin an identity $e$ to the topological algebra $A$ [Naimark (1959), p. 170] and 
thereby obtain a topological algebra $A+e$ in which (2.5) is equivalent to

$$
(e-f) *(e-g)=e,
$$

so that $f$ has a quasi-inverse if and only if $e-f$ has an inverse. Through (2.6) we can show that a quasi-inverse is unique, so that, when it exists, we can write $f^{\prime}$ for the quasi-inverse of $f$.

For any $f, g, h$ in $A$ with $f=g+h$ we can define formally

$$
k=h *(e-g)^{-1}=h *\left(e-g^{\prime}\right)=h-h * g^{\prime},
$$

the last expression for which is in $A$ whenever $g^{\prime}$ exists; and we can calculate formally

$$
\begin{aligned}
-f^{\prime} & =(e-g-h)^{-1}-e=(e-g-k *(e-g))^{-1}-e \\
& =(e-g)^{-1} *(e-k)^{-1}-e=-g^{\prime}-k^{\prime}+g^{\prime} * k^{\prime},
\end{aligned}
$$

the last expression for which is in $A$ whenever $k^{\prime}$ exists. These identities are used rigorously in the following arguments, which settle immediately the properties of quasi-inverses.

LemMA 1. For each $f$ in $A$ there exists a unique quasi-inverse $f^{\prime}$.

Proof. The uniqueness of $f^{\prime}$ follows algebraically from its existence, to prove which we choose $a>0$ such that $\int_{0}^{a}|f(x)| d x<1$ and we define $h(x)=f(x)-g(x)$ on $[0,+\infty)$, where

$$
\begin{aligned}
g(x) & =f(x) & & \text { on }[0, a), \\
& =0 & & \text { on }[a,+\infty) .
\end{aligned}
$$

Then $g$ is in $L^{1}(0,+\infty)$, which is a Banach algebra under the compositions of $A$; and $\|g\|_{1}<1$, so that $g^{\prime}=-\sum_{n=1}^{\infty} g^{* n}$ converges in $L^{1}(0,+\infty)$ to a quasi-inverse of $g$. Thus $k$ is defined by (2.7) as an element of $A$. Moreover $h(x)=k(x)=0$ on $[0, a)$, so that $h^{* n}(x)=k^{* n}(x)=0$ on $[0, n a)$; and thus $-\sum_{n=1}^{\infty} k^{* n}$ converges in $(A, U)$ to a quasi-inverse of $k$. It follows by direct multiplication that (2.8) defines a quasi-inverse of $f$.

THEOREM 1. The map $f \rightarrow f^{\prime}$ is a homeomorphism from $(A, T)$ onto $(A, T)$.

Proof. Since $f \rightarrow f^{\prime}$ is its own inverse, we need only show it is continuous near any $g$ in $A$. For any $r=1,2, \cdots$ we thus let $b=t_{r}\left(g^{\prime}\right)$ and note by (2.7) that if $f=g+h$ then $t_{r}(k) \leqq(1+b) t_{r}(h)$. We now choose $h$ so small that $(1+b) t_{r}(h)<1$, and show by $(2.4)$ and $(2.8)$ that

$$
\begin{aligned}
t_{r}\left(f^{\prime}-g^{\prime}\right) \leqq & (1+b) t_{r}\left(k^{\prime}\right), \\
& \text { where } t_{r}\left(k^{\prime}\right) \leqq(1+b) t_{r}(h)_{i}^{\prime}\left[1-(1+b) t_{r}(h)\right] .
\end{aligned}
$$


This theorem implies that $A$ is a ring with continuous quasi-inverse, and that $A+e$ is a ring with continuous inverse [Naimark (1959), pp. 170-175]. Each element of $A+e$ has the form $c e+f$ with $c$ in $C, f$ in $A$; and each $c e+f$ has an inverse in $A+e$ if and only if $c \neq 0$. (Of course each nonzero $f$ has an inverse in the Mikusinski field of generalized functions [Mikusinski (1959), Chapter 3].) The following stronger result will be needed in the next section.

Lemma 2. For any $f$ in $A$ the series $\sum_{n=1}^{\infty} f^{* n}$ converges in $T$ to- $f^{\prime}$.

Proof. Define $a, g, h$ as in Lemma 1 and $k$ as in (2.7). Then the functions now listed have $T$-convergent series in powers $g^{* r} * h^{* s}$ with nonnegative coefficients: $-g^{\prime}$ by construction, $k$ by $(2.7),-k^{\prime}$ by construction, and $-f^{\prime}$ by (2.8). Moreover the $T$-convergent series for $-|f|^{\prime}$ in terms of $|g|$ and $|h|$ dominates the corresponding series for $-f^{\prime}$ in terms of $g$ and $h$, since

$$
\left|\left[g^{* r} * h^{* s}\right](x)\right| \leqq\left[|g|^{* r} *|h|^{* s}\right](x) \quad \text { for } r, s=1,2, \cdots
$$

However this series for $-|f|^{\prime}$ converges absolutely almost everywhere, because its terms are all nonnegative; so that the series for $-f^{\prime}$ converges absolutely almost everywhere, and its terms can be arbitrarily rearranged. In particular, the rearranged series

$$
\sum_{n=1}^{\infty} f^{* n}=\sum_{n=1}^{\infty} \sum_{m=0}^{n}\left(\begin{array}{l}
n \\
m
\end{array}\right) g^{* m} * h^{* n-m}
$$

converges almost everywhere to $-f^{\prime}$ and is bounded by $-|f|^{\prime}$, hence converges in $T$ to $-f^{\prime}$ by the dominated convergence theorem.

3. Integral equations. The equation (1.1)-(1.2) in the notation just introduced becomes

$$
v=f+g * v
$$

so that the $n$th Picard approximation from the starting point $v_{0}$ is

$$
v_{n}=f * \sum_{m=0}^{n-1} g^{* m}+v_{0} * g^{* n} \text { for } n=0,1,2, \cdots,
$$

where $g^{* 0}=e$. If $|g|^{k}$ is locally integrable for some $k>1$ then $h=g^{* l}$ is locally bounded for large enough $l$ by the Riesz convexity theorem [Dunford and Schwartz (1958), p. 528, Exercise 7], and

$$
\left|h^{* n}(x)\right| \leqq u_{r}(h)^{n} \cdot x^{n-1} /(n-1) ! \quad \text { almost everywhere on }[0, r)
$$


for $n=1,2, \cdots$ by induction. For any $m=0,1, \cdots$ we can factor $g^{* m}=$ $g^{* p} * h^{* q}$ with $0 \leqq p<l$, and estimate

$$
u_{r}\left(f * g^{* m}\right) \leqq t_{r}\left(f * g^{* p}\right) u_{r}(h)^{q} r^{q-1} /(q-1) !
$$

by (2.4) and (3.3). Thus $\left\{v_{n}\right\}$ converges in the topology $U$ whenever $h$ is locally bounded, and $\left\{v_{n}\right\}$ converges to a continuous function whenever $f, g, v_{0}$ are continuous.

However there exist functions $g$ in $L^{1}(0,+\infty)$ for which no $|g|^{k}$ with $k>1$ is integrable near 0 and for which all $g^{* l}$ with $l=1,2, \cdots$ are unbounded near 0 [Feller (1971), p. 516], so that $\left\{v_{n}\right\}$ need not converge uniformly on any interval containing 0 . Thus the following theorem should be of interest.

THEOREM 2. For any $f$ and $g$ in $A$ the equation (3.1) has the unique solution $v=f-f * g^{\prime}$, which depends continuously on $f$ and $g$ in the topology $T$. For any $v_{0}$ in $A$ the Picard sequence $\left\{v_{n}\right\}$ converges to $v$ in the topology $T$.

Proof. If $v$ is any solution of (3.1) then by Theorem 1

$$
v=(e-g)^{-1} *(v-g * v)=\left(e-g^{\prime}\right) * f=f-f * g^{\prime} ;
$$

conversely this last expression satisfies (3.1) and depends continuously on $f$ and $g$. As $n \rightarrow \infty$ the powers $g^{* n}$ converge $(T)$ to 0 by Lemma 2, and the sums $f * \sum_{m=0}^{n-1} g^{* m}$ converge $(T)$ to $f-f * g^{\prime}$, so that the functions $v_{n}$ converge $(T)$ to $f-f * g^{\prime}$.

The quasi-inverse $g^{\prime}$ is essentially the resolvent kernel for (3.1) [Tricomi (1957), p. 8], and certain investigations require that this kernel be in $L^{1}(0,+\infty)$ (e.g. [Miller (1968)]). Thus we abbreviate $L=L^{1}(0,+\infty)$ and we define

$$
L^{\prime}=\left\{f \in A: f^{\prime} \in L\right\} .
$$

We wish to describe $L^{\prime}$ in both the topology $T$ and the stronger topology $V$ given by the metric $d(f, g)=\|f-g\|_{1}$ (or equivalently by $d^{\prime}=d /(1+d)$ if we wish to avoid infinite distances).

THEOREM 3. L' $L^{\prime}$ is a set of first category for the topology $T$, and is an open subset of $A$ for the topology $V$.

Proof. By Theorem 1 the subsets $L$ and $L^{\prime}$ of $(A, T)$ have the same topological properties. However if $B_{r}=\left\{f \in A: t_{r}(f) \leqq 1\right\}$ then $L=$ $\bigcup_{n=1}^{\infty} n \bigcap_{r=1}^{\infty} B_{r}$, so that $L$ is a countable union of sets closed in $A$, each having void interior. For any $g$ in $L^{\prime}$ the inequality (2.10) holds with $r=$ $+\infty$, that is, with $t_{r}(f)=\|f\|_{1}$, so that some $V$-neighborhood of $g$ is in $L^{\prime}$.

Given a complex polynomial $P(z)=\sum_{m=1}^{n} p_{m} z^{m}$ we can define a corresponding polynomial $P^{*}(f)=\sum_{m=1}^{n} p_{m} f^{* m}$ such that $P(z) \rightarrow P^{*}(f)$ is an 
algebra homomorphism for each fixed $f$ in $A$. However Theorem 1 of this development states that $A$ is a radical ring [NaImark (1959), p. 164], whence the functional calculus for topological algebras shows that the preceding homomorphism extends to all functions $P(z)$ analytic at the origin [Waelbroeck (1954)]. That is, if $P(z)=\sum_{m=1}^{\infty} p_{m} z^{m}$ converges in any neighborhood of the origin, then $P^{*}(f)=\sum_{m=1}^{\infty} P_{m} f^{* m}$ converges in $T$ for any $f$ in $A$. This remark yields the existence of a solution for a nonlinear integral equation such as

$$
\sum_{m=1}^{n} p_{m} v^{* m}=P^{*}(v)=f, \quad \text { with } p_{1} \neq 0 .
$$

Another radical algebra has been used recently by [Grabiner (1970)] to study integral equations containing finite convolutions. by

Each $f$ in the subalgebra $L^{1}[0,+\infty)$ has a Laplace transform $L f$ given

$$
L f(s)=\int_{0}^{\infty} \exp (-s x) f(x) d x \quad \text { on } \operatorname{Re}(s) \geqq 0 .
$$

The mapping $f \rightarrow L f$, by the convolution theorem, takes Laplace convolution into pointwise multiplication, hence defines a homomorphism from $L^{1}[0,+\infty)$ into some function algebra with pointwise compositions on $\operatorname{Re}(s) \geqq 0$. This transform can be extended variously to larger domains (e.g. [Krabbe (1966)]), and equation (3.1) can be solved thus in some cases. However transform methods are less general than our results, in that the mapping $f \rightarrow L f(s)$ cannot be extended to a nontrivial homomorphism on all of $A$. Indeed if $q$ is a homomorphism from $A$ into $C$, and if $q(f)=c \neq 0$ for some $f$ in $A$, then $q(g)=1$ for $g=f / c$, and

$$
q\left(g^{\prime}\right)=q\left(g * g^{\prime}\right)=q\left(g+g^{\prime}\right)=1+q\left(g^{\prime}\right),
$$

which is impossible, so that $q \equiv 0$.

ACKnowledgment. The author would like to thank Professor R. K. Miller for his comments on this work and the referee for his suggestions to improve it.

\section{REFERENCES}

N. Dunford and J. T. Schwartz, Linear operators. I: General theory, Pure and Appl. Math., vol. 7, Interscience, New York, 1958. MR 22 \#8302.

W. Feller, An introduction to probability theory and its applications. Vol. II, 2nd ed., Wiley, New York, 1971. MR 42 \#5292.

S. Grabiner, The use of formal power series to solve finite convolution integral equations, J. Math. Anal. Appl. 30 (1970), 415-419. MR 41 \#4153.

G. Krabbe, Ratios of Laplace transforms, Mikusinski operational calculus, Math. Ann. 162 (1965/66), 237-245. MR 32 \#6165.

W. V. Lovitt, Linear integral equations, McGraw-Hill, New York, 1924. 
J. G. Mikusiński, Operational calculus, Internat. Series of Monographs on Pure and Appl. Math., vol. 8, Pergamon Press, New York; PWN, Warsaw, 1959. MR 21 \#4333.

R. K. Miller, On Volterra integral equations with nonnegative integrable resolvents, J. Math. Anal. Appl. 22 (1968), 319-340. MR 37 \#3291.

R. K. Miller and A. Feldstein, Smoothness of solutions of Volterra integral equations with weakly singular kernels, SIAM J. Math. Anal. 2 (1971), 242-258.

M. A. Naìmark, Normed rings, GITTL, Moscow, 1956; English transl., Noordhoff, Groningen, 1959. MR 19, 870; MR 22 \#1824.

H. H. Schaefer, Topological vector spaces, Macmillan, New York, 1966. MR 33 \#1689.

F. G. Tricomi, Integral equations, Pure and Appl. Math., vol. 5, Interscience, New York, 1957. MR 20 \#1177.

L. Waelbroeck, Le calcul symbolique dans les algèbres commutatives, C. R. Acad. Sci. Paris 238 (1954), 556-558; J. Math. Pures Appl. 33 (1954), 147-186. MR 17, 512; 513.

Mathematical Sciences Department, iBM Thomas J. Watson Research Center, YoRKTOWN HeIGHTS, NEW YORK 10598 